\title{
Application of ALE to nonlinear wave radiation by a non-wall-sided structure
}

\author{
by Jie Zhang*, Student Member Masashi Kashiwagi*, Member
}

\begin{abstract}
Summary
Water waves generated by the forced oscillation of a 3D non-wall-sided structure are analyzed based on the fully nonlinear potential-flow theory. In order to track an exact position of the free surface, Arbitrary Lagrangian-Eulerian (ALE) scheme is used in the computation. The feature of ALE scheme is that by introducing a prescribed path (curve) for each fluid marker on the free surface, the movement of fluid marker representing the deformation of free surface is confined along a path. With properly-designed configuration of those paths, this scheme brings about several advantages compared with the Mixed Eulerian-Lagrangian scheme and the Semi-Lagrangian scheme which are extensively used. In the computation, a higher-order boundary element method (HOBEM) and the 4th-order Runge-Kutta method are adopted as the solver of an initial boundary value problem (BVP). In the validation, the radiated waves generated by a truncated circular cylinder with flare oscillating in heave or surge are studied. Regarding the calculation of hydrodynamic forces, the temporal derivative of the velocity potential $\left(\phi_{t}\right)$ is evaluated in an exact but simple manner, i.e. $\phi_{t}$ is obtained by solving a reconstructed BVP without evaluating second-order derivatives of the velocity potential. Besides hydrodynamic forces, wave profiles at specific points and wave run-ups are also computed. Comparisons of the present results against published results of corresponding computations are made. To evaluate capability of the proposed ALE scheme, bodies with curved large flare are used in the computation.
\end{abstract}

\section{Introduction}

In ocean engineering, for the problems that no wave breaking and flow separation exist or viscosity and strong free-surface nonlinearities are confined in a small flow region, the numerical simulation based on the fully nonlinear potential theory (FNPT) is still an economical choice. In the context of FNPT, the existence of flare poses challenge in the computation. Compared to a structure with wall-sided geometry near the waterline, the flare could cause rapid variation in the fluid velocity and pressure and thus good mesh quality is required near the body throughout the computation. In addition, a numerical treatment without taking account of body geometry above the still waterline may lead to a spurious intersection after one time step in the computation, for instance, the intersection may pierce the body surface, see the right-hand side of Fig. 1. In general, once the body moves with rotational motion, even the body without flare encounters the same problem. Furthermore, those problems become more prominent when the body oscillates with large amplitude, where the mesh may be compressed or stretched to a large extent. As a consequence, quality of the mesh near the body would deteriorate and affect the accuracy and stability of the computation.

In connection with the problem mentioned above, to develop a robust and accurate scheme for capturing the free surface, we should consider the following two conditions: (1) the intersection should be captured and updated exactly; (2) the mesh on the free surface should be self-adaptive in order to conform to the body motion or complex geometry above the still waterline. With those

* Graduate School of Engineering, Osaka University Received 12 May 2017

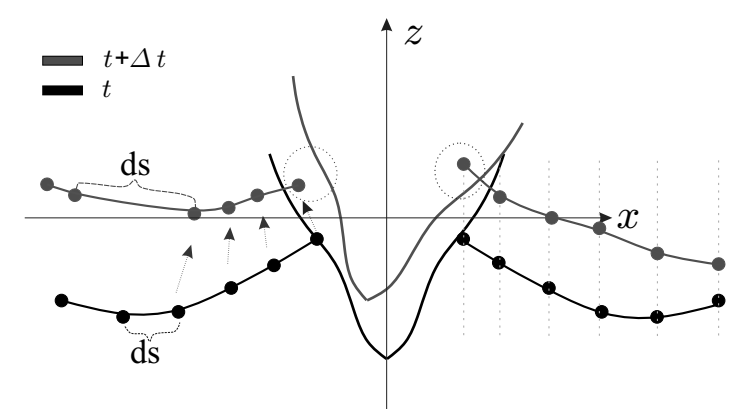

Lagrangian method Semi-Lagrangian method

Fig. 1 Illustration of numerical problems in free surface mesh management near a body which has complex geometry and/or oscillates with large amplitude.

requirements in mind, the existing schemes are reviewed first.

The so-called Mixed-Eulerian-Lagrangian (MEL) scheme proposed by Longuet-Higgins $\&$ Cokelet $^{1)}$ has been extensively used in the literature due to several advantages, such as easy implementation and capability of dealing with overturning waves. However, due to the nature of this scheme that a fluid particle moves with much freedom, the arrangement of fluid particles becomes irregular and hence we need to reconstruct the mesh, see the left-hand side of Fig. 1. Thus some modifications should be made, when applying to wave-body interaction problems. In order to meet condition (1) mentioned above, Shirakura \& Tanizawa ${ }^{2}$ and Liu, Xue $\& Y^{3} e^{3)}$ derived an ALE-type formulation for updating the intersection point. For avoiding a situation that the distance between two neighboring fluid particles becomes too close or too far, a regridding scheme is applied frequently, which is related to condi- 
tion (2). However, the regridding scheme as an artificial procedure would cause loss of the information of fluid flow.

Alternatively, the Semi-Lagrangian scheme ${ }^{4)}(\mathrm{SL})$ is also widely used in wave-body interaction problems, which is illustrated in the right-hand side of Fig. 1. In this scheme, the horizontal motion of fluid marker is fixed in the horizontal direction, while vertically the marker moves freely in order to represent the deformation of the free surface. In other words, the fluid marker on the free surface only moves along a vertical path. Compared to the MEL scheme, the SL scheme need not use a regridding scheme because of a fact that the horizontal distance between two neighboring markers is time-invariant (condition (2)). However a drawback $^{6)}$ of this scheme is that it is applicable only to cases when the body surface is vertical near the waterline (condition (1)).

A comparative study of different schemes for tracking the free surface may be summarized as follows. The excessive freedom of Lagrangian fluid particle makes the MEL scheme capable to handle complex deformation of the free surface, but the fluid particle must be relocated frequently to dismiss unwanted motion of the particle. On the other hand, relocation of the fluid marker is avoided in the SL scheme by restricting the horizontal motion of the fluid marker at the sacrifice of flexibility to treat a body with large flare.

After the review described above, a logical question arises. Is there an optimized compromise between MEL and SL schemes, where advantages of both methods are reserved while disadvantages are minimized? The answer is positive. The starting point for realizing this optimized compromise is to increase flexibility of the SL scheme. In order to take into account a complex body geometry above the waterline, a curved path analogous to the body's local geometry is introduced for each fluid marker on the free surface, where the movement of the marker along this path represents the free surface deformation. On the other hand, in order to adapt to a large-amplitude motion of the body, the prescribed path may also translate and/or rotate in connection with the body motion. The idea of this method is illustrated in Fig. 2. By carefully designing these parameters, i.e. the shape, motion, and arrangement of these paths, the mesh on the free surface can be well controlled. Detailed discussion on this method would be introduced later. Since the inherent idea of this method is taken from the $\mathrm{ALE}^{5)}$ scheme, this scheme will be referred to as the ALE scheme hereafter.

When studying the sloshing in a non-wall-sided tank, Zhang ${ }^{6}$ ) also encountered those problems mentioned above. In his research, a modified Semi-Lagrangian method is proposed, where the idea is very similar to the present ALE scheme, however the starting point for derivation is largely different, which would be discussed later. What is more, the present ALE scheme is applicable to more practical cases where the flare is of arbitrary shape.

Another issue in the computation of nonlinear wave-body interactions is how to properly evaluate the temporal derivative of the velocity potential $\phi_{t}$. Although $\phi_{t}$ can be evaluated accurately by a post-processing procedure in the radiation problem, the final goal of the present study is to establish a robust and accurate method which can simulate large-amplitude motions of a 3-D freely floating body in waves. Thus, $\phi_{t}$ must be computed accurately at each time instant for hydrodynamic forces and body motions to provide the velocity and position of the body. As pointed out by Tanizawa ${ }^{7)}$ $\phi_{t}$ is related to the acceleration potential and an exact BVP can be

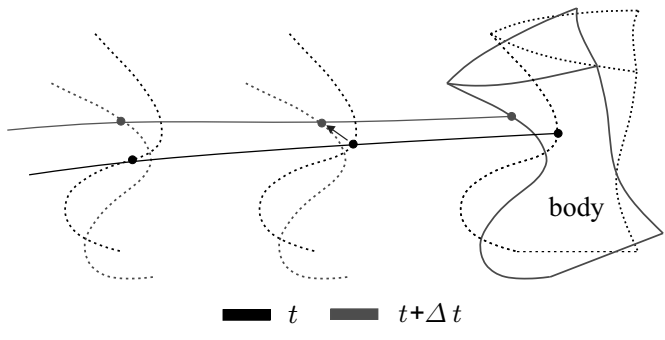

Fig. 2 Sketch of ALE procedure.

set up for directly solving this term. However, the body-surface boundary condition of this BVP includes second spatial derivatives of the velocity potential. To evaluate this term also needs information on the curvature on a body, see Kashiwagi ${ }^{8)}$ in the 2D computation. In 3D computations, the expression for calculating this term is very much complicated, see Berkvens ${ }^{9)}$ and Shirakura \& Tanizawa $^{2}$. On the other hand, Wu \& Eatock Taylor ${ }^{10)}$ devised an indirect method to evaluate the hydrodynamic force, and an expression including second derivatives is transformed into a simpler form by using Stokes' theorem, where the application of this method can be found in Zhou \& Ning, et al ${ }^{11)}$ and Bai $\&$ Taylor ${ }^{12)}$. In the present research, $\phi_{t}$ is directly evaluated by solving a reconstructed BVP without requirement of calculating the second derivatives ${ }^{13)}$. Zhang $^{6)}$ also used this formulation in a sloshing problem.

In the present paper, computed time histories of the wave profile are compared with the results published by other researchers. The hydrodynamic forces are computed by three-point central difference method (post-processing method) and by solving a BVP. Both methods agree well with each other. The time histories of hydrodynamic forces are decomposed by Fourier-series analysis up to third-order components. The first-harmonic component associated with the added mass and damping coefficient is compared with the corresponding results obtained by an independent frequency-domain method. In order to verify capability of the proposed ALE scheme, bodies with curved large flare near the waterline are used in the computation.

\section{Basic Equations}

\section{1 Boundary value problem}

We define a Cartesian coordinate system $o-x y z$ so that the $z$ axis of the system is positive upwards and the origin is on the undisturbed free surface. The fluid is assumed to be inviscid and incompressible, and the flow is irrotational. The velocity potential $\phi$ can therefore be introduced, which satisfies the Laplace equation in the fluid domain,

$$
\nabla^{2} \phi=0
$$

and is subject to various boundary conditions on the entire boundary surface $S$ of the fluid domain.

On the instantaneous free surface $S_{F}$, the kinematic and dynamic conditions can be written as

$$
\left\{\begin{array}{l}
\frac{\partial \eta}{\partial t}=\frac{\partial \phi}{\partial z}-\nabla \phi \cdot \nabla \eta \\
\frac{\partial \phi}{\partial t}=-g \eta-\frac{1}{2} \nabla \phi \cdot \nabla \phi-\frac{P}{\rho}
\end{array}\right.
$$


where $z=\eta(x, y, t)$ is the free surface elevation; $g$ the gravitational acceleration; $\rho$ the density of water; $P$ the atmospheric pressure which is set to zero in the paper. The kinematic boundary condition on the instantaneous wetted body surface $S_{B}$ is

$$
\frac{\partial \phi}{\partial n}=\boldsymbol{V} \cdot \boldsymbol{n}
$$

where $\boldsymbol{n}$ is the unit normal vector on the body surface pointing out of fluid domain and $\boldsymbol{V}$ is the velocity of a point on the body. Appropriate boundary conditions are also necessary on a control surface $S_{C}$ far from the body to avoid unwanted wave reflection. In the present study, an artificial damping layer is used to absorb the wave energy of radiated wave, which will be described later.

\section{2 ALE scheme}

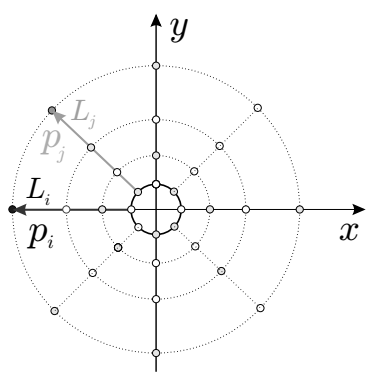

a.

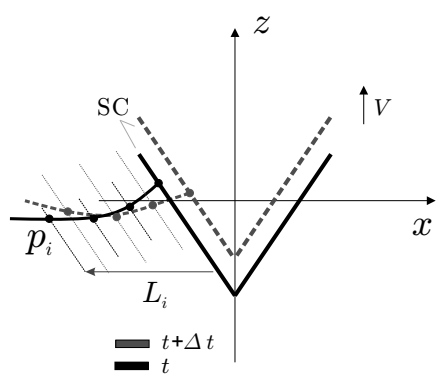

b.
Fig. 3 Illustration of ALE scheme, where radiated waves are generated by a heaving circular cone. a. top view of initial mesh of free surface; $b$. side view of computation domain.

As explained above, the feature of ALE scheme is to introduce a prescribed path for each node on the free surface. The associated problems are;

(i) how to design an appropriate path for each fluid marker;

(ii) how to collocate all of those paths in space, where at least the path should not intersect with each other;

(iii) how to optimize the motion (translation and/or rotation) of each path in order to adapt to large-amplitude motion of the body.

In terms of problem (i), in the present research, the path is generated by translation of sectional line on the body, see Fig. 2. More specifically, the path of point $p_{i}$ illustrated in Fig. 3 is generated by translation of body's sectional line $(S C)$ along a stationary vector (in most cases) $\boldsymbol{L}_{i}$. The way of determining this vector $\boldsymbol{L}_{i}$ would be discussed later. This strategy requires us only to define and store very few curves as paths for all fluid markers.

Because the prescribed path servers as the trajectory of fluid marker, too close or too far distance of two paths directly affects the quality of the free surface mesh. This problem is related to problem (ii) and can be resolved at the initial stage, where the free surface is discretized by a fine mesh at the beginning as seen in Fig. 3 a. At this stage, each fluid marker is provided with a unique vector (e.g. $\boldsymbol{L}_{i}$ of $p_{i}$ ), indicating that the path of $p_{i}$ would always keep its distance and orientation from the body surface. By this way, the relative position of neighboring fluid markers is locked and thus the aspect ratio of each element on the free surface is time-invariant. This strategy retains good topology of initial mesh throughout the long-term simulation.

Regarding problem (iii), as we can see in Fig. 3 b, the body's motion may increase the projected free-surface area on the horizontal plane, and the mesh near this region would be stretched if there is no proper adjustment. In order to make the mesh selfadjustable with the body's motion, all paths in the ALE scheme are defined to move in parallel to the sectional line of the body. By doing so, the mesh near the waterline can be self-adjustable when a body is in large-amplitude motion. Furthermore, since all nodes on the free surface move uniformly following the body's motion, this strategy enables us to simulate the motions with large horizontal displacement, e.g. maneuvering motion of a ship, in terms of limited computational domain and mesh.

Taking account of those considerations mentioned above for the ALE scheme, the position of prescribed path and the sectional line of a body can be related by the following relations:

$$
\left\{\begin{array}{l}
\boldsymbol{x}_{p}=\boldsymbol{x}_{p^{\prime}}+\boldsymbol{L}_{p} \\
\dot{\boldsymbol{x}}_{p}=\dot{\boldsymbol{x}}_{p^{\prime}}+\dot{\boldsymbol{L}}_{p}
\end{array}\right.
$$

where $\boldsymbol{x}_{p}$ is an arbitrary point on the prescribed path and $\boldsymbol{x}_{p^{\prime}}$ the corresponding point on body's sectional line, where $\boldsymbol{x}_{p}$ and $\boldsymbol{x}_{p^{\prime}}$ coincide once the prescribed path is translated back towards $-\boldsymbol{L}_{p}$. Note that if there is no yaw motion, $\dot{\boldsymbol{L}}_{p}=0$, otherwise $\boldsymbol{L}_{p}$ would also rotate about the $z$ axis. Fig. 4 illustrates this mapping between $p$ and $p^{\prime}$.

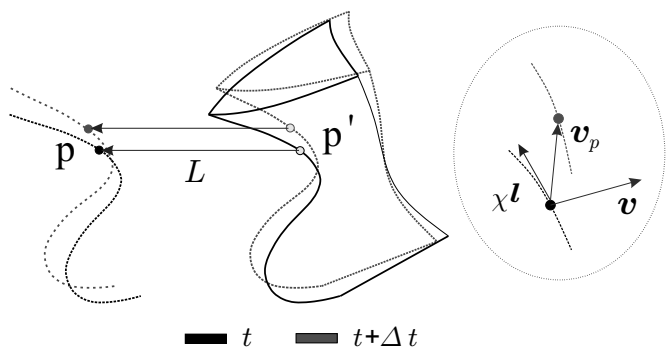

Fig. 4 Mapping between prescribed path and body's sectional line.

Once the problem mentioned above has been solved, the subsequent problem is how to determine the new position of a fluid marker moving along a curve which is also in motion. Under this circumstance, two constraint conditions should be imposed on the motion of fluid marker; (a) the velocity of marker $\boldsymbol{V}_{p}$ should be parallel to the tangential vector of the path, see Fig. 4; (b) $\boldsymbol{V}_{p}$ should also be subject to the kinematic boundary condition on the free surface. According to condition (a), $\boldsymbol{V}_{p}$ can be expressed as follows:

$$
\boldsymbol{V}_{p}=\chi \boldsymbol{l}+\boldsymbol{V}
$$

where $\boldsymbol{l}$ is the tangential vector of prescribed path at point $P, \chi$ a scalar and $\boldsymbol{V}$ the velocity at point $P$ due to the motion of path itself. Note that the path is approximated by cubic-spline and $\boldsymbol{l}$ can be evaluated numerically for an arbitrary body geometry.

Substitution of Eq. (5) into the kinematic boundary condition 
of the free surface and rather lengthy transformation yields,

$$
\chi=\frac{\nabla \phi \cdot \nabla(z-\eta)-\boldsymbol{V} \cdot \nabla(z-\eta)-\mu \eta}{\nabla(z-\eta) \cdot \boldsymbol{l}}
$$

where $\phi$ stands for the velocity potential; $\eta$ the wave elevation with $z=\eta(x, y, t)$ on the free surface; and $\mu$ the artificial damping for avoiding the wave reflection at a far field. A more detailed derivation can be found in Zhang and Kashiwagi ${ }^{14)}$, where the ALE scheme is applied for solving the nonlinear wave diffraction.

Substituting Eqs. (5) and (6) into the dynamic free-surface condition, we have

$$
\frac{\delta \phi}{\delta t}=-g \eta-\frac{1}{2}(\nabla \phi)^{2}+\boldsymbol{V}_{p} \cdot \nabla \phi-\mu \phi
$$

where $\frac{\delta}{\delta t}=\frac{\partial}{\partial t}+\boldsymbol{V}_{\boldsymbol{p}} \cdot \nabla$ denotes the material derivative, following a marker on a moving path.

Equations (5), (6) and (7) provide the ALE-type free-surface conditions and can be used further for time stepping. Compared to the MEL and SL schemes, these free-surface conditions contain terms of $\boldsymbol{V}$ and $\boldsymbol{l}$, indicating that both motion and geometry of the body are taken into account in the proposed ALE scheme.

In comparison to the MEL and SL schemes, the advantages of this ALE approach are highlighted here. First, the curved path which fits closely with body's local geometry can detect easily the position of a fluid marker above the waterline; motion of the path as a response to large-amplitude motion of the body makes selfadjustment of the mesh possible, which is crucial for long-term simulation. Second, the mesh on the free surface needs to be generated only once at the beginning, and the mesh can be generated automatically in subsequent times without relocation of the fluid markers. In addition, this approach is very efficient in implementation, since we need only to restore $N 3 \mathrm{D}$ curves represented by the cubic spline for general cases that a body has complex geometry, where $N$ is the number of nodes along the waterline.

As mentioned before, Zhang ${ }^{6)}$ also developed a modified SL (MSL) scheme for a study on the sloshing in a non-wall-sided tank. However, there is an important difference in this scheme. In the MSL scheme a coordinate transformation is adopted such that the new $z^{\prime}$ axis is set to be parallel to the inclined wall, and thus the fluid marker only moves 'vertically' in the $z$ ' direction. In the derivation in this paper, a material derivative is used with very clear physical meaning. Furthermore, because the prescribed path is represented by a parametric curve ${ }^{14)}$ in the present study, the intersection can be updated exactly even for a very complex body geometry. On the contrary, it seems that the MSL scheme is only applicable to very simple cases, where the flare is inclined, i.e. the tangential vector of the flare is constant.

\section{3 Pressure and hydrodynamic forces}

In order to obtain hydrodynamic forces and moments acting on the body, first we need to consider a solution of $\phi_{t}$ appearing in Bernoulli's pressure equation. When a body has only the translational motion, $\phi_{t}$ is subject to the following $\mathrm{BVP}^{10)}$

$$
\left\{\begin{array}{l}
\nabla^{2} \phi_{t}=0 \\
\frac{\partial \phi}{\partial t}=-\frac{1}{2}(\nabla \phi)^{2}-g z \quad \text { on } S_{F} \\
\frac{\partial \phi_{t}}{\partial n}=\dot{\boldsymbol{U}} \cdot \boldsymbol{n}-\boldsymbol{U} \cdot \frac{\partial \nabla \phi}{\partial n} \quad \text { on } S_{B}
\end{array}\right.
$$

As we can see, there is a second derivative term $\partial \nabla \phi / \partial n$ appearing in the body boundary condition. Directly evaluating this term requires much effort in a 3D problem, see Berkvens ${ }^{9}$ and Shirakura \& Tanizawa ${ }^{2)}$. In order to circumvent this difficulty, following $\mathrm{Wu} \& \mathrm{Hu}^{13)}$ and $\mathrm{Zhang}^{6)}$, we introduce an auxiliary function, $\psi=\phi_{t}+\boldsymbol{U} \cdot \nabla \phi$. The BVP for $\psi$ can be rewritten as follows:

$$
\left\{\begin{array}{l}
\nabla^{2} \psi=0 \\
\psi=-\frac{1}{2}(\nabla \phi)^{2}-g z-\boldsymbol{U} \cdot \nabla \phi \quad \text { on } S_{F} \\
\frac{\partial \psi}{\partial n}=\dot{\boldsymbol{U}} \cdot \boldsymbol{n} \quad \text { on } S_{B}
\end{array}\right.
$$

Once $\psi$ is obtained, the pressure and resulting hydrodynamic forces and moments can be computed directly from Bernoulli's pressure equation.

However, in the present radiation problem, where hydrodynamic forces and body motions are decoupled, $\phi_{t}$ can be approximated by using a finite difference scheme after time series of $\phi$ is obtained (post-processing method). Specifically, by denoting the velocity potential of the collocation point $i$ on the body surface (see Fig. 5) at time $t$ as $\phi_{i}(t)$ and the velocity of point $i$ as $\boldsymbol{v}_{\boldsymbol{i}}, d \phi_{i} / d t$ can be written as

$$
\frac{d \phi_{i}(t)}{d t}=\frac{\partial \phi_{i}(t)}{\partial t}+v_{i} \cdot \nabla \phi_{i}(t)
$$

In this post-processing method, $d \phi_{i} /\left.d t\right|_{t=t_{0}}$ could be evaluated by a central finite difference in terms of the information at $t=$ $t_{0}+n \Delta t$ and $t=t_{0}-n \Delta t$. In this paper, $d \phi_{i} /\left.d t\right|_{t=t_{0}}$ is evaluated by the three-point central difference scheme, and thus

$$
\left.\frac{\partial \phi_{i}(t)}{\partial t}\right|_{t=t_{0}}=\frac{\phi_{i}\left(t_{0}+\Delta t\right)-\phi_{i}\left(t_{0}-\Delta t\right)}{2 \Delta t}-\boldsymbol{v}_{i}\left(t_{0}\right) \cdot \nabla \phi_{i}\left(t_{0}\right)
$$

Note that $\boldsymbol{v}_{\boldsymbol{i}}$ equals neither the body velocity nor $\nabla \phi$, because of mesh movement. However, it can be evaluated in a similar manner to that for $d \phi_{i} / d t$, since the trajectory of node $i$ is known as shown in Fig. 5.

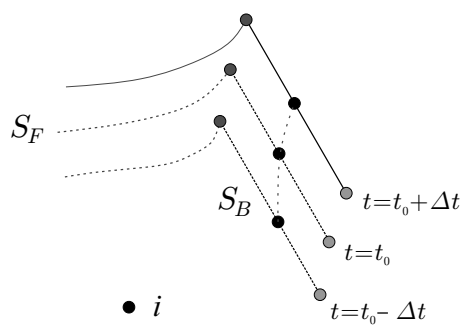

Fig. 5 Evolution of free surface and wetted body surface.

\section{Numerical Solution Procedure}

\section{1 HOBEM}

In the BVP proposed in the preceding section, application of Green's second identity leads to the following equation for evaluation of the unknowns:

$$
\begin{aligned}
C(\boldsymbol{x}) \phi(\boldsymbol{x}) & -\iint_{S} \phi_{n}(\boldsymbol{\xi}) G(\boldsymbol{x} ; \boldsymbol{\xi}) d S(\boldsymbol{\xi}) \\
& +\iint_{S} \phi(\boldsymbol{\xi}) G_{n}(\boldsymbol{x} ; \boldsymbol{\xi}) d S(\boldsymbol{\xi})=0
\end{aligned}
$$


where $G(\boldsymbol{x} ; \boldsymbol{\xi})=1 /|\boldsymbol{x}-\boldsymbol{\xi}|$ is the Rankine source Green function, with $\boldsymbol{x}$ the field point and $\boldsymbol{\xi}$ the source point; $C(\boldsymbol{x})$ is the solid angle at field point $\boldsymbol{x}$, which can be evaluated by the following equation:

$$
C(\boldsymbol{x})=-\iint_{S} \frac{\partial G(\boldsymbol{x} ; \boldsymbol{\xi})}{\partial n} d S(\boldsymbol{\xi})
$$

Equation (12) is solved by a higher-order boundary element method (HOBEM), where the boundary surface $S$ is discretized into curvilinear quadrilaterals with nine nodes. Specifically, the bi-quadratic isoparametric interpolation is used in HOBEM to represent both boundary surface and unknowns on the boundary.

The discretized form of Eq. (12) can be written as

$$
\left[\begin{array}{ll}
A_{1} & A_{2}
\end{array}\right]\left\{\begin{array}{c}
\left.\phi\right|_{S_{B}} \\
\left.\frac{\partial \phi}{\partial n}\right|_{S_{F}}
\end{array}\right\}=\left[\begin{array}{ll}
C_{1} & C_{2}
\end{array}\right]\left\{\begin{array}{c}
\left.\frac{\partial \phi}{\partial n}\right|_{S_{B}} \\
\left.\phi\right|_{S_{F}}
\end{array}\right\}
$$

where the influence coefficients $A_{1}, A_{2}$ and $C_{1}, C_{2}$ in the matrices in Eq. (14) are computed with HOBEM. The velocity potential on the body surface and the normal velocity on the free surface are unknowns; these are actually their values at nodal points of quadratic panels and can be determined by solving the boundary integral equation, Eq. (14). After these are determined, the velocity potential and position of the free surface are then updated from Eqs. (5)-(7).

\section{2 Other noteworthy numerical techniques}

In Eq. (6), there is a term containing $\nabla \eta$, which can be evaluated in general by interpolation. However, $\nabla \eta$ is related to the normal vector of the free surface and hence can be evaluated directly, once the normal vector of the free surface is known. Recalling the definition of the unit normal vector on the free surface

$$
\left(n_{1}, n_{2}, n_{3}\right)=\frac{\left(-\eta_{x},-\eta_{y}, 1\right)}{\sqrt{\left(1+\eta_{x}^{2}+\eta_{y}^{2}\right)}},
$$

$\nabla \eta$ can be expressed as

$$
\eta_{x}=-\frac{n_{1}}{n_{3}}, \quad \eta_{y}=-\frac{n_{2}}{n_{3}}
$$

An artificial damping layer is imposed on the truncated boundary of a far field in order to avoid unwanted wave reflection. Following Bai \& Eatock Taylor ${ }^{12)}$, the strength of artificial damping can be written as follows:

$$
\mu(R)= \begin{cases}\alpha \omega \frac{\left(R-R_{0}\right)^{2}}{(\beta \lambda)^{2}}, & R_{0} \leq R \leq R_{D} \\ 0, & R<R_{0}\end{cases}
$$

where $\omega$ is the circular frequency of incident wave; $R_{0}$ the radius of starting point of the damping zone; $R_{D}$ the radius of outer boundary; and $\alpha$ and $\beta$ are the coefficients to control the strength and length of the damping zone, see Fig. 7. In the present study, $\alpha$ and $\beta$ are set equal to 1.0 .

In order to allow a gradual development of the numerical solutions, the amplitude of oscillation is modulated by the following function:

$$
M(t)= \begin{cases}\frac{1}{2}\left\{1-\cos \left(\frac{\pi t}{T_{m}}\right)\right\}, & t \leq T_{m} \\ 1, & t>T_{m}\end{cases}
$$

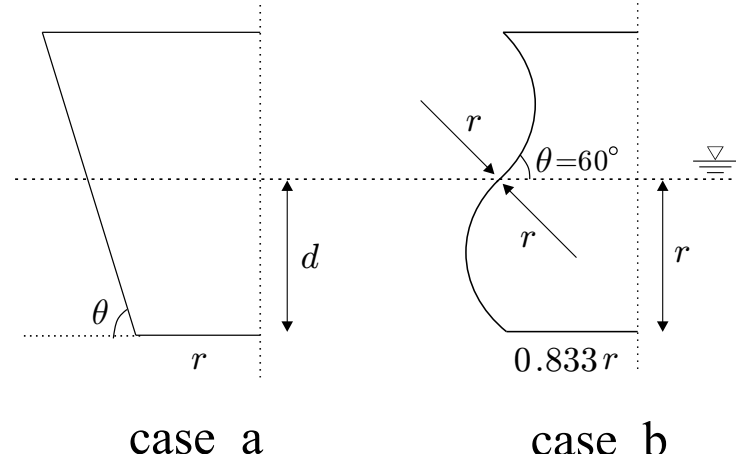

Fig. 6 Dimension of the axisymmetric body with flare; a. body with inclined flare and $b$. body with curved flare.

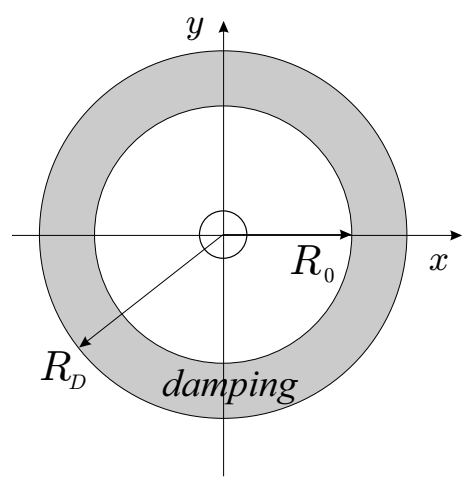

Fig. 7 Sketch of damping layer.

where $T_{m}$ is the period of modulation and chosen as $2 T$ in the present computation.

In addition, to suppress the so-called saw-tooth instability, a smoothing scheme introduced by Koo \& $\mathrm{Kim}^{4}$ ) is used, which is applied every 10 time steps. In the present computation, the 4th-order Runge-Kutta method is used for time integration and the GMRES algorithm is used for solving a linear system of simultaneous equations. The so-called double-node technique is applied on the corners such as an intersection point between the body and the free surface. Because the wetted body surface is timedependent, at each time step body surface should be remeshed, where the detailed procedure on remeshing can be found in in Zhang and Kashiwagi ${ }^{14)}$.

\section{Numerical Results and Discussions}

We consider the radiation problem for a non-wall-sided cylinder shown in Fig. 6, forcibly oscillating the body in open sea with infinite water depth. Note that the geometry of case b in Fig. 6 is very relevant to a practical application, e.g. the geometry near the ship's bulbous bow, and to handle this kind of geometry is tricky in the computation as we will see later. In the simulations below, the initial draft of the cylinder is set to $d=1.5 r$ in case $a$. The cylinder is subject to the following harmonic motion in the vertical or 
horizontal direction:

$$
Z=A \sin (\omega t) \quad \text { or } \quad X=A \sin (\omega t)
$$

The computational domain is truncated by a vertical circular cylinder with radius $R_{D}=3 \lambda$, where $\lambda$ is the wave length computed by the linear theory. The length of damping zone is set equal to $\lambda$ as shown in Fig. 7.

\section{1 Convergence study}

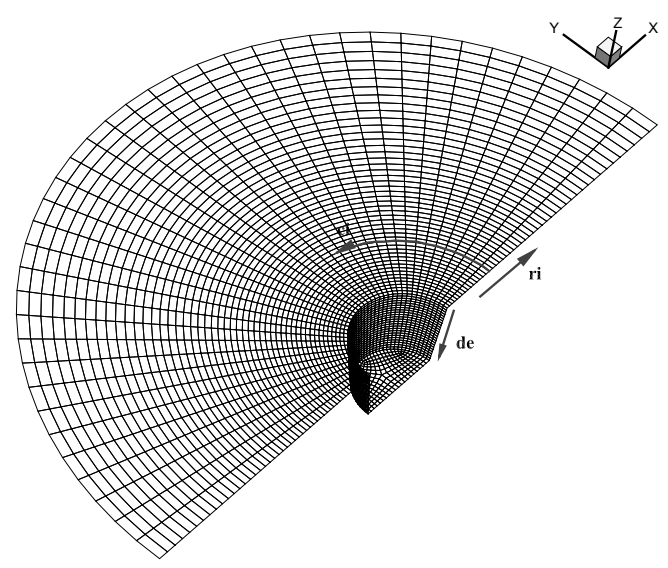

Fig. 8 Sketch of mesh1 used for convergence test. ri denotes radial direction; $c i$ the direction along azimuth angle and de the direction along draft. On bottom, unstructured mesh is used.

In the convergence tests, the wave radiation around a circular cylinder with $\theta=80^{\circ}$ (case $a$ ) under prescribed heave motion is considered. The wavenumber is taken as $k r=2.42$ and the amplitude of motion as $A / r=0.4$. Three different meshes are chosen, as shown in Table 1 and mesh1 is illustrated in Fig. 8 for reference. On the free surface, along the radial direction (ri) 17, 20, and 24 nodes per wave length are used in mesh1, mesh2, and mesh3, respectively. From Fig. 9, we can see the results are sensitive to the number of mesh, especially in the force, which is expected since higher-order force components are affected by the mesh. However, the results obtained from mesh 2 and mesh 3 are substantially identical, indicating that the present computation is converged in terms of mesh discretization.

Figure 10 illustrates results of convergence study in terms of time step. As we can see, even if a larger time step is used, e.g. $\Delta t=T / 60$, there is no noticeable discrepancy on the results, which proves sufficient convergence of the present results in terms of time discretization. In the subsequent computations, the free surface is

Table 1 Details of different meshes, nodes in direction- $1 \times$ nodes in direction-2, or total nodes.

\begin{tabular}{|c|c|c|c|}
\hline Item & free surface (ri $\times$ ci) & side surface $($ de $\times$ ci) & bottom \\
\hline \hline mesh1 & $52 \times 40$ & $18 \times 40$ & 214 \\
\hline mesh2 & $60 \times 56$ & $20 \times 56$ & 476 \\
\hline mesh3 & $72 \times 60$ & $24 \times 60$ & 827 \\
\hline
\end{tabular}

discretized by 20 nodes per wave length if not specified, and the discretization in other directions can be approximated by keeping a proper aspect ratio of the mesh, and $\Delta t=T / 80$ is used.

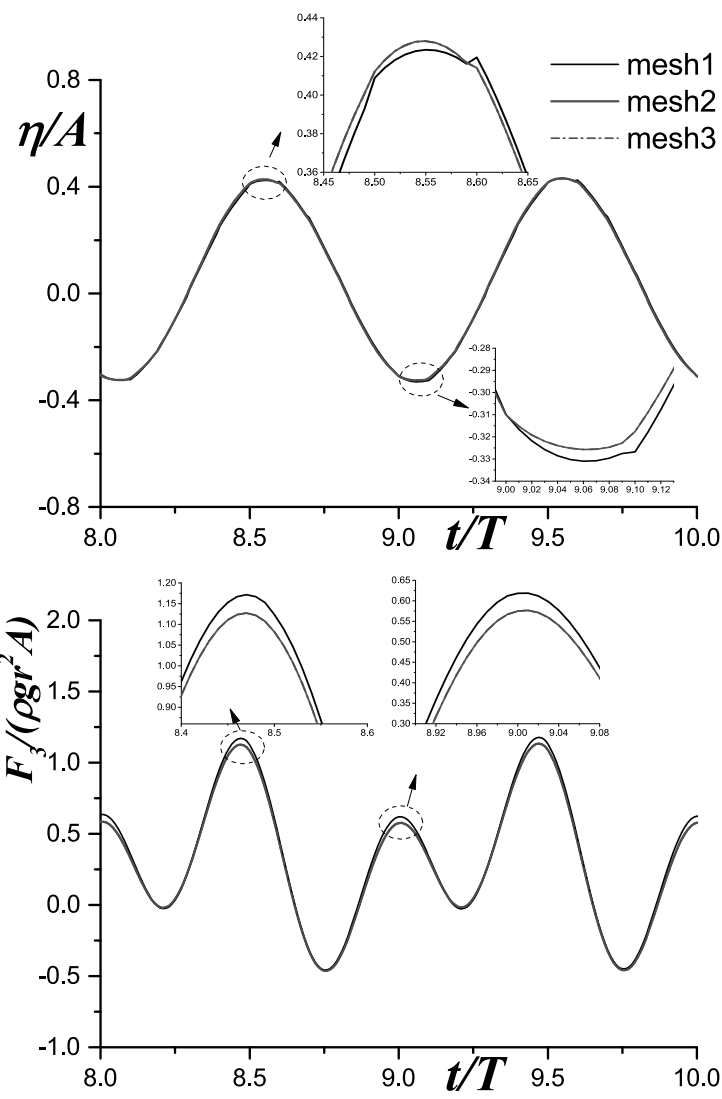

Fig. 9 Study on mesh-dependency of wave run up and vertical force, with $\Delta t=T / 100$.

\section{2 Wave radiation due to heave motion, case a}

We first consider a cylinder (case $a$ ) with flare angle of $\theta=$ $80^{\circ}$, which is in heave motion with amplitude of $A=0.1 \mathrm{r}$ and $A=0.4 r$ and non-dimensional wavenumber of $k r=0.64$ and $k r=$ 1.95. The wave run-up and the vertical force are calculated and the results are shown in Fig. 11 for $k r=0.64$ and Fig. 12 for $k r=$ 1.95. In computed results of the vertical force, $B V P$ indicates that $\phi_{t}$ is evaluated by solving a BVP while Diff indicates that $\phi_{t}$ is approximated by the three-point central finite difference. We can see that both methods give identical results, which shows validity of solving $\phi_{t}$ by Eq. (9). From the aspect of efficiency, evaluation of $\phi_{t}$ by solving a BVP would not increase the computation time, since the coefficient matrix for $\phi_{t}$ is exactly the same as that for $\phi$. In the present paper, since the validity of the method for computing $\phi_{t}$ has been confirmed through comparison of the results by two different methods, hereafter we will show only the results of the hydrodynamic force calculated by solving Eq. (9).

We can see also from Fig. 12 at higher frequency that both wave run-up and hydrodynamic force exhibit nonlinearity with increasing the oscillation amplitude.

In order to validate computed results more, a comparison is made with the results by Wang $\& \mathrm{Wu}^{15)}$ for the wave elevation on 

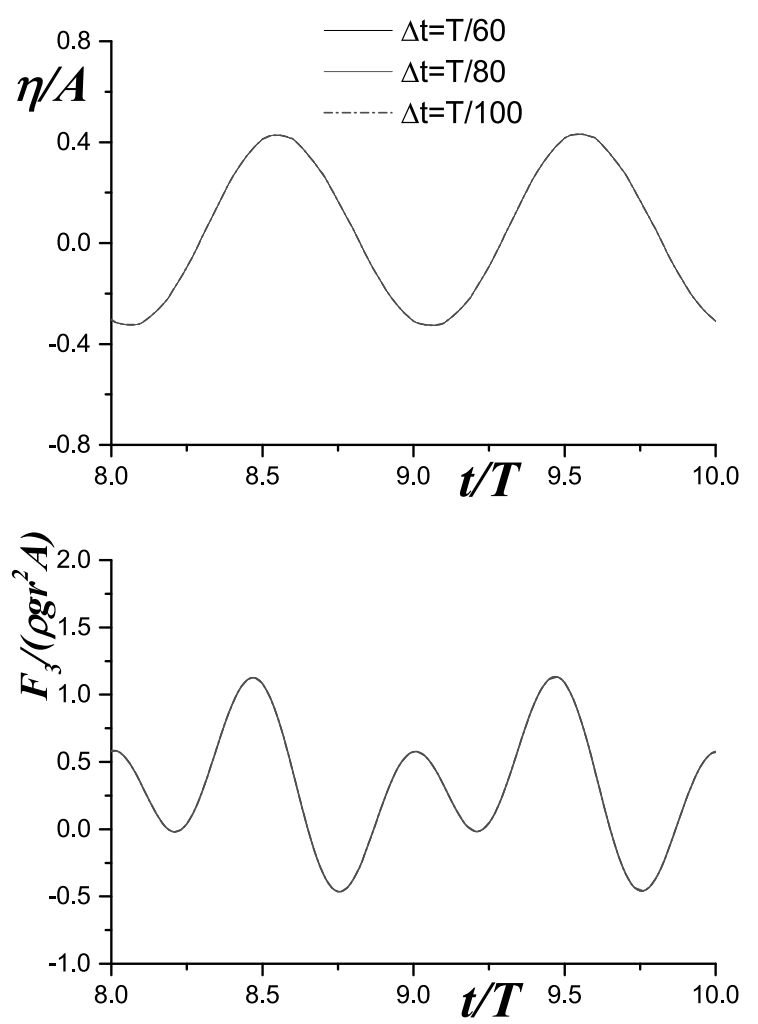

Fig. 10 Study on time-step-dependency of wave run up and vertical force. mesh2 is used in the computation.
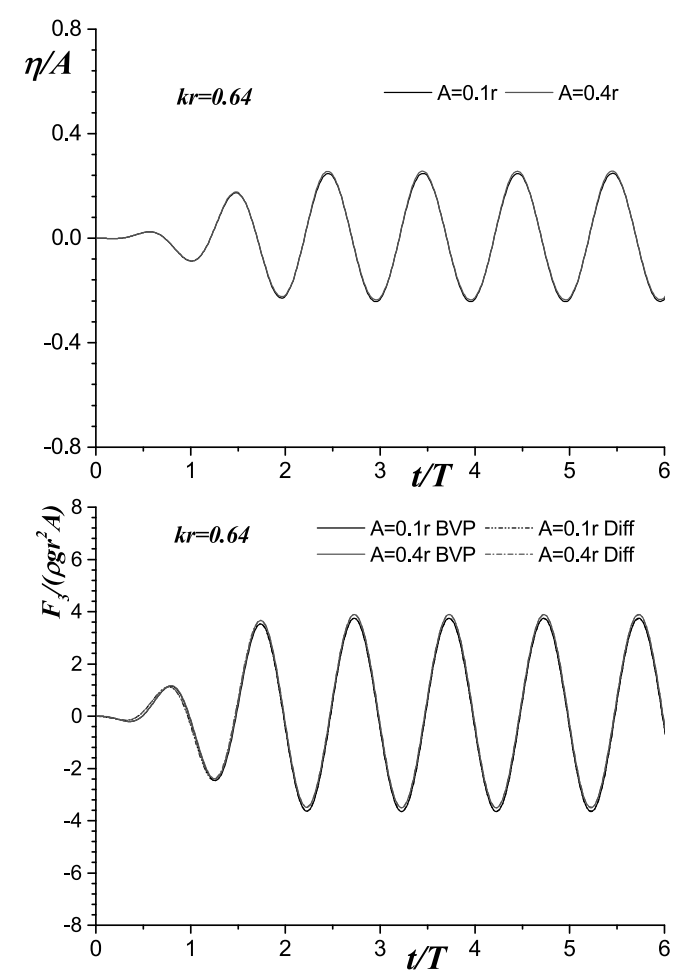

Fig. 11 Time histories of wave run-up and vertical force by forced heave oscillation at $K r=0.64$.
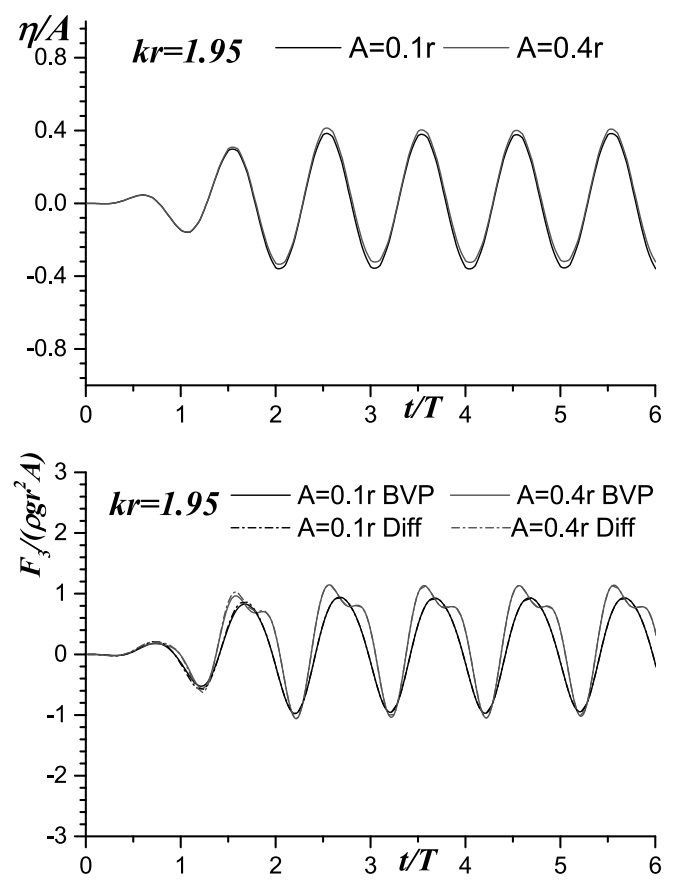

Fig. 12 Time histories of wave run-up and vertical force by forced heave oscillation at $K r=1.95$.

the free surface at $R=2 r$. Computed results are shown for two cases: (1) $k r=1$ and $A / r=0.6$ and (2) $k r=2$ and $A / r=0.3$. As we can see from Fig. 13, the present results agree well with those by Wang $\& \mathrm{Wu}^{15)}$. This may indicate that the velocity field is properly solved.

According to Bernoulli's pressure equation, the hydrodynamic force consists of three components, i.e. the component computed from $\phi_{t}$, the component due to the velocity squared, and the static force. In order to validate the present results for the force calculation, comparison is again made with the results by Wang \& $\mathrm{Wu}^{15}$ ) for each of the components. As we can see from Fig. 14, the agreement in all three components is good. By looking at the order of each component in the force and the total force, we can see that different force components may cancel out and as a consequence

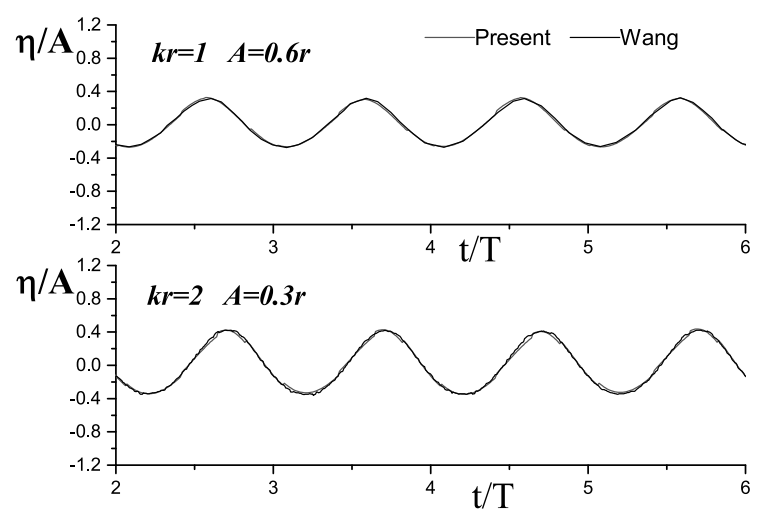

Fig. 13 Time histories of wave elevation at $R=2 r$ on free surface at $k r=1, A=0.6 r$ and $k r=2, A=0.3 r$. 
the total force does not exhibit apparent nonlinearity.

The Fourier analysis on the time history of hydrodynamic forces is carried out. The hydrodynamic force acting in the $i$-th direction, $F_{i}$, was represented by the following Fourier series:

$$
\begin{aligned}
F_{i} & =-\rho \iint\left\{\frac{\partial \phi}{\partial t}+\frac{1}{2}(\nabla \phi)^{2}\right\} n_{i} d S \\
& =F_{i}^{(0)}+\sum_{n=1}^{5}\left\{a^{(n)} \sin (n \omega t)+b^{(n)} \cos (n \omega t)\right\}
\end{aligned}
$$

The first-order force is expressed in terms of the added mass and the damping coefficient. In the case of forced heave oscillation $(i=3)$, those quantities are nondimensionalized as follows:

$$
\left\{\begin{array}{l}
A_{33}=a^{(1)} /\left(-A \omega^{2}\right) /\left(\rho r^{3}\right) \\
B_{33}=b^{(1)} /(A \omega) /\left(\rho r^{2} \sqrt{g r}\right)
\end{array}\right.
$$

The remaining $n$ th-harmonic components are nondimensionalized in the following form ${ }^{8)}$

$$
F_{i}^{(0)}=F_{i}^{(0)} /\left(\rho g r^{3}(A / r)^{2}\right), \quad F_{i}^{(n)}=F_{i}^{(n)} /\left(\rho g r^{3}(A / r)^{n}\right)
$$

where $F_{i}^{(n)}=\sqrt{a^{(n)^{2}}+b^{(n)^{2}}}$ is the amplitude and the phase can be computed from $\delta^{(n)}=\arctan \left(b^{(n)} / a^{(n)}\right)$.
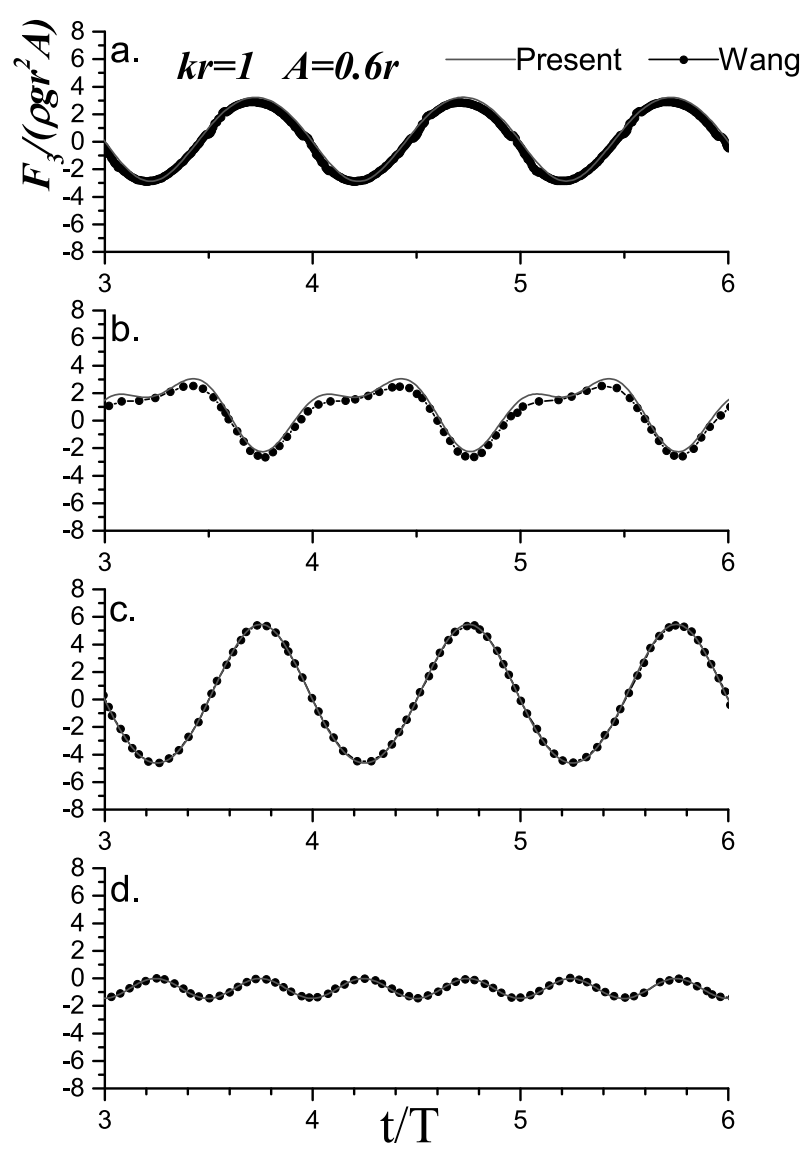

Fig. 14 Time history of non-dimensional force at $k r=1$ and $A=0.6 r$; a. vertical force in total; $\mathrm{b}$. force component due to $\phi_{t} ; \mathrm{c}$. force component due to $g z$; d. force component due to $1 / 2(\nabla \phi)^{2}$.
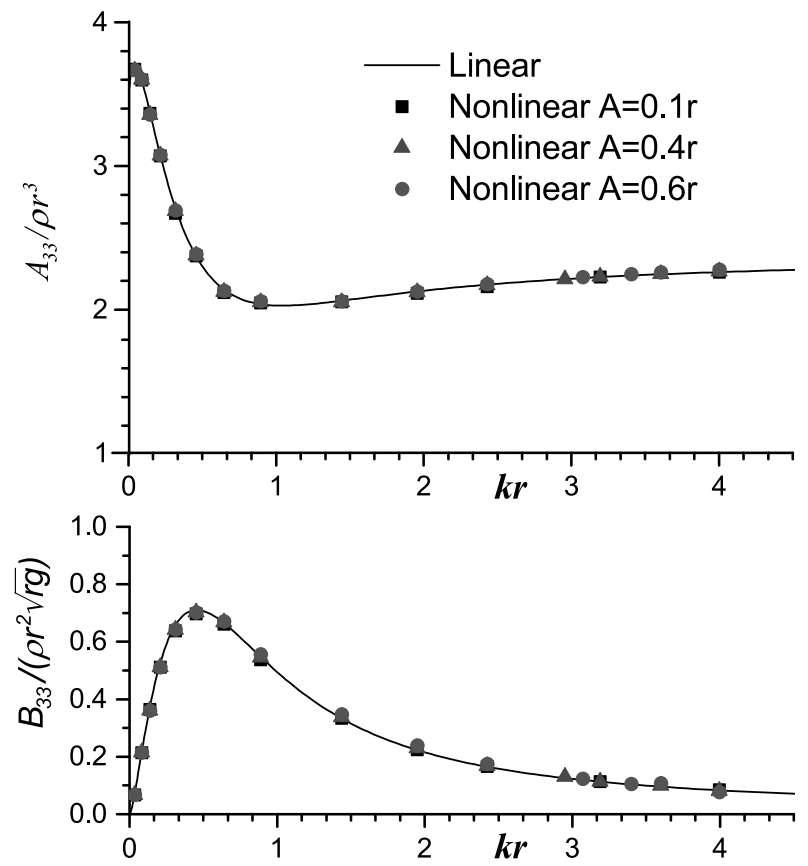

Fig. 15 Added mass and damping coefficient of a heaving cylinder with flare in different amplitudes.

Figure 15 illustrates the added mass and the damping coefficient of a heaving flared body at three different oscillation amplitudes. From comparison with linear results computed by HydroSTAR, the present results agree well with the linear results and no amplitude-dependency is found. This indicates that the firstorder hydrodynamic forces are little influenced by the variation of wetted body surface and the nonlinear free-surface condition as far as the heave motion of the present body is concerned.

Figures 16 and 17 show the 2nd-order and 3rd-order hydrodynamic forces, respectively. Looking at the 2nd-order forces, both amplitude and phase of the harmonic component at two different oscillation amplitudes are the same in the nondimensional value divided by the oscillation amplitude squared. On the other hand, slight difference can be seen in the 2nd-order steady-force component at higher frequencies.

On the other hand, in the 3rd-order hydrodynamic forces, obvious differences depending on the oscillation amplitude can be observed. Particularly the phase is different even in the low frequency range. We should note that the amplitude of the 3rd-order hydrodynamic force for the present body is small as compared to the 2nd-order force and thus the small difference in the amplitude tends to be shown exaggeratedly.

The influence of the flare on wave run-ups and hydrodynamic forces is studied by changing the angle of the flare at $\theta=85^{\circ}$, $\theta=80^{\circ}$ and $\theta=75^{\circ}$. The computed results are given in Fig. 18 and Fig. 19 for $k r=1$ and $k r=2$, respectively, but with the same amplitude $A=0.6 r$. These computed results clearly indicate that the larger flare, the larger wave run-up and vertical force. Furthermore from comparison between Fig. 18 and Fig. 19, we can see that nonlinearity in the vertical force becomes prominent at higher frequencies. However, the time variation in the wave run-up looks sinusoidal and the increase in the amplitude seems to be propor- 


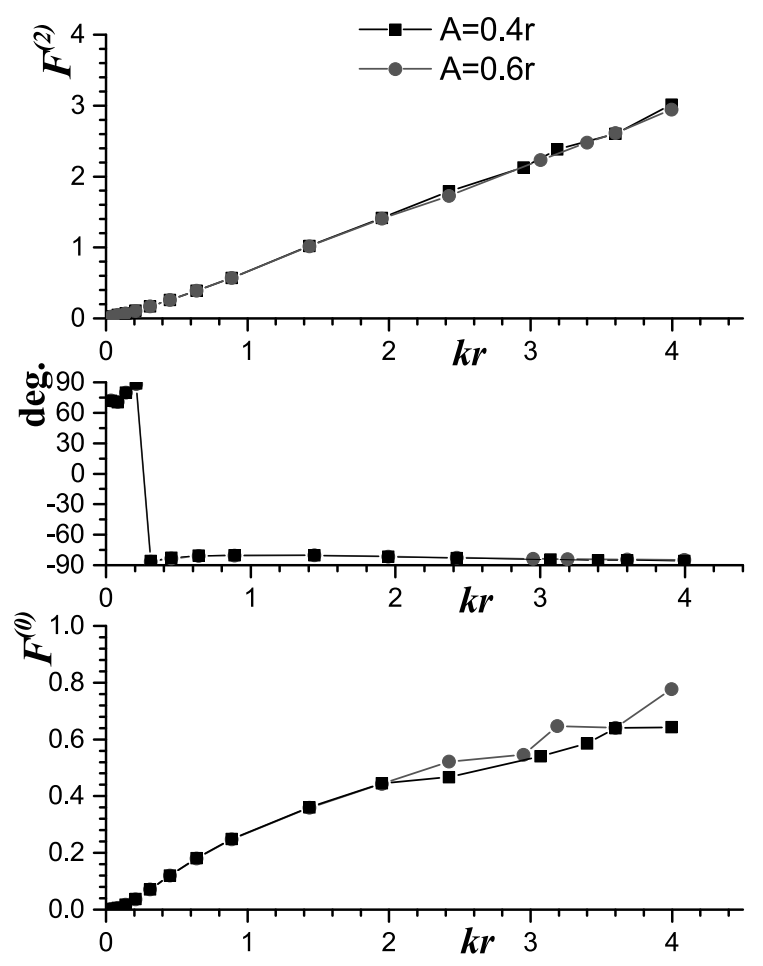

Fig. 16 Non-dimensional amplitude of 2nd-harmonic force as well as phase with $A=0.4 r$ and $A=0.6 r$.

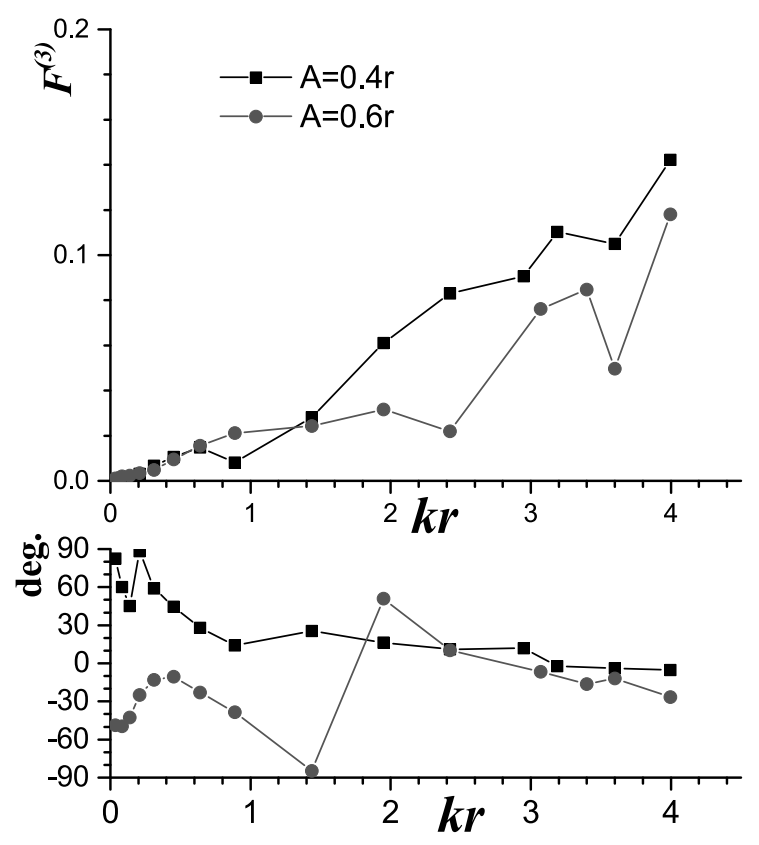

Fig. 17 Non-dimensional amplitude of 3rd-harmonic force as well as phase with $A=0.4 r$ and and $A=0.6 r$.

tional to the increase in the flare angle. Existence of a flare makes the body more blunt and thus at the same oscillation amplitude, a body with larger flare could generate waves with larger amplitude. The computed results presented here are consistent with the

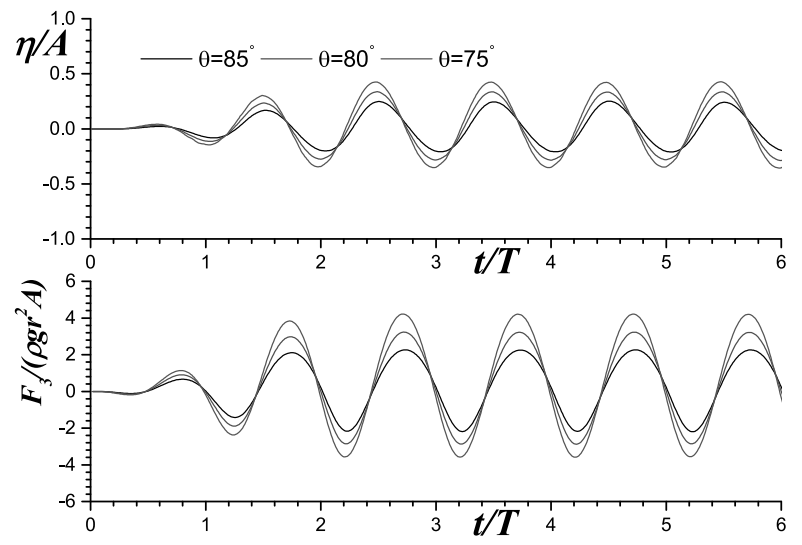

Fig. 18 Flare effect on wave run-up and vertical force with $A=0.6 r$ and $k r=1$.

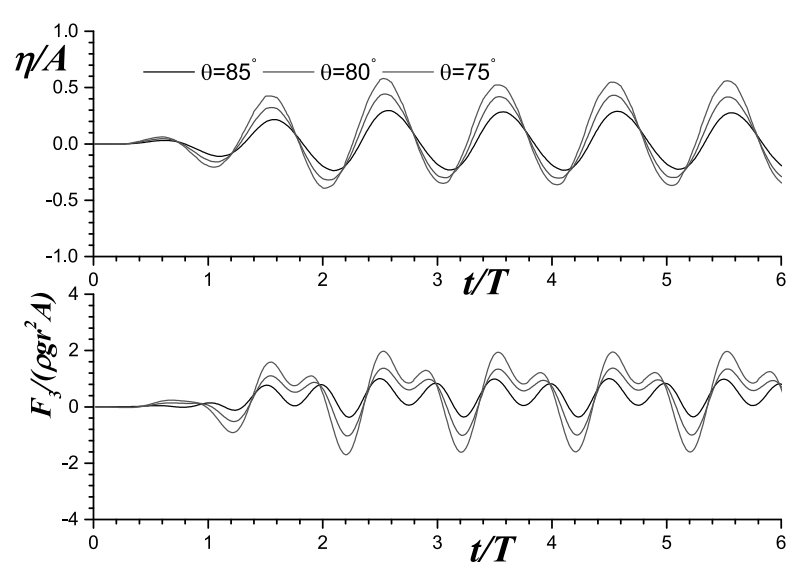

Fig. 19 Flare effect on wave run-up and vertical force with $A=0.6 r$ and $k r=2$.

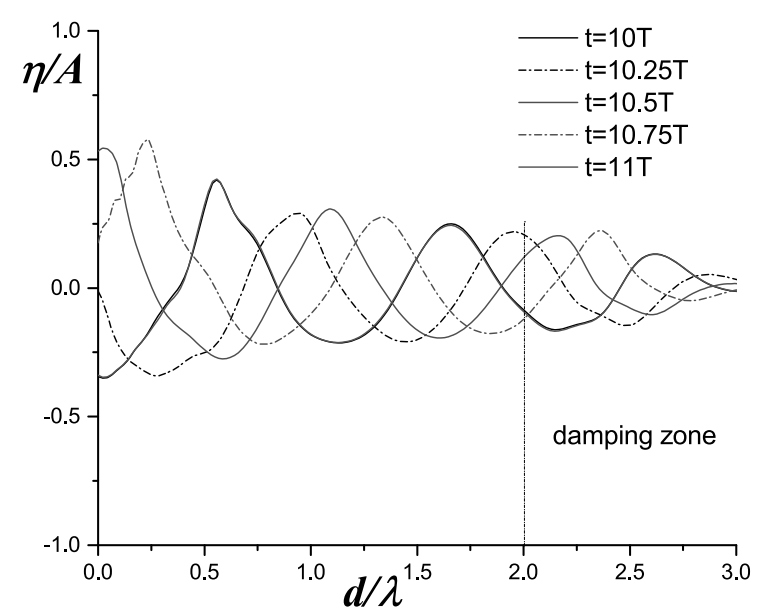

Fig. 20 Wave profile along radial direction of the computational domain ( $d$ is distance from body) with $A=0.6 r, k r=2$, and $\theta=75^{\circ}$. 


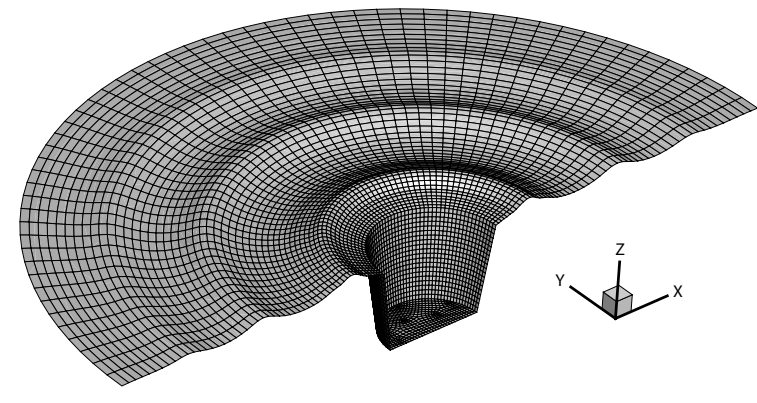

Fig. 21 Sketch of wave radiation by a heaving cylinder with flare, at $t=11.45 T$ with $k r=3.6$ and $A=0.6 r$

computation shown by others ${ }^{15)}$.

In order to investigate the performance of artificial damping layer, the wave profile along the radial direction of the domain is given in Fig. 20. As we can see, the wave profile at $t=11 T$ looks identical to that at $t=10 T$, which implies that no wave reflection from the truncated outer boundary is observed. This result also indicates that a smoothing scheme adopted in this paper extracts very little energy from the wave. On the other hand, these spatial wave profiles also exhibit apparent nonlinearity especially near the body surface. Fig. 21 gives a snapshot of the body-generated wave at $t=11.45 T$ for $k r=3.6$ and $A=0.6 r$.

\section{3 Wave radiation due to heave motion, case $b$}

In order to confirm capability of the proposed ALE scheme, the radiation wave generated by an axisymmetric body is studied, see case $b$ in Fig. 6 . As can be seen, the geometry of this body has rapid variation near the waterline, which implies that a fine mesh and smaller time step should be used in the computation to capture small-scale wave phenomena as well as rapid variation in the velocity and pressure. In the present case, the time step is set equal to $\Delta=T / 100$ and a smoothing scheme is applied every five time steps.

Computed results including the time history of the wave runup and vertical force are provided for $k r=1.147$ and $k r=2.294$. The results at $k r=1.147$ are provided with three heaving amplitudes, i.e. $A / r=0.1, A / r=0.2$, and $A / r=0.3$, for comparison. However, since the wave at $k r=2.294$ was found very violent near the waterline, only two oscillation amplitudes are used, i.e. $A / r=0.1$ and $A / r=0.2$.

From Figs. 22 and 23, we can see that a high-frequency oscillation becomes prominent with increase of the amplitude in the time history of the wave run-up, which is due to geometrical nonlinearity that the flare makes the wave-body intersection move largely and hence the curved orbit of the motion of intersection together with nonlinear local-wave effects does contribute to the fluctuation in the wave run-up. Another possible reason might be a numerical error, e.g. the generation of so-called sawtooth wave. However, the time history of the hydrodynamic force is smooth with apparent nonlinearity, indicating that the local nonlinearity affects the global force to some extent.

In order to further investigate the source of nonlinearity in the time history of the force, the force components corresponding to each terrm in Bernoulli's pressure equation are shown in Fig. 24.
From this comparison, we can see that the hydrodynamic force is dominant in the case of $k r=2.294$ while the static force is the largest in magnitude in the case of $k r=1.47$; which is consistent
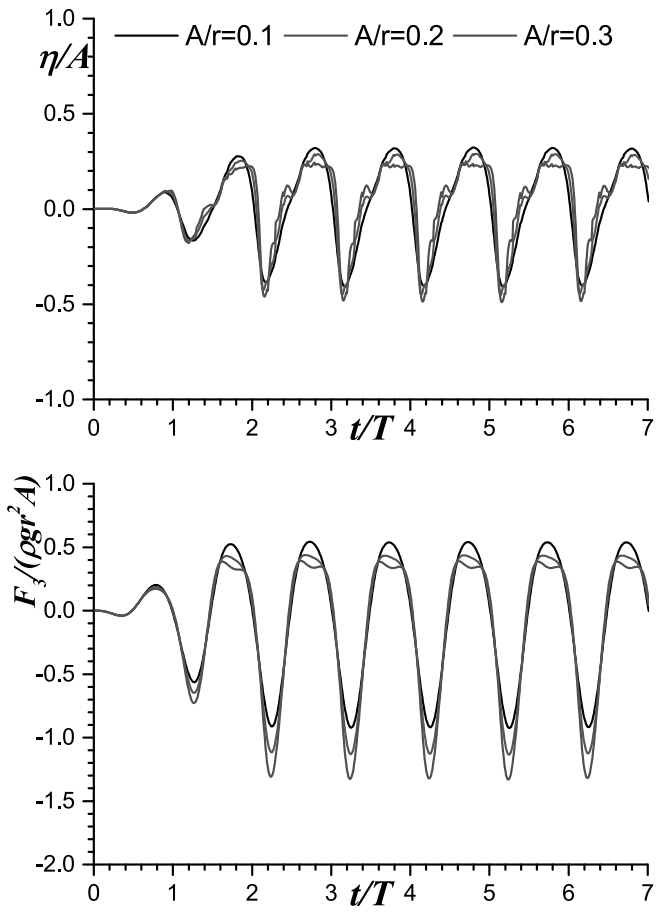

Fig. 22 Time history of wave run up and vertical force with $k r=1.147$.
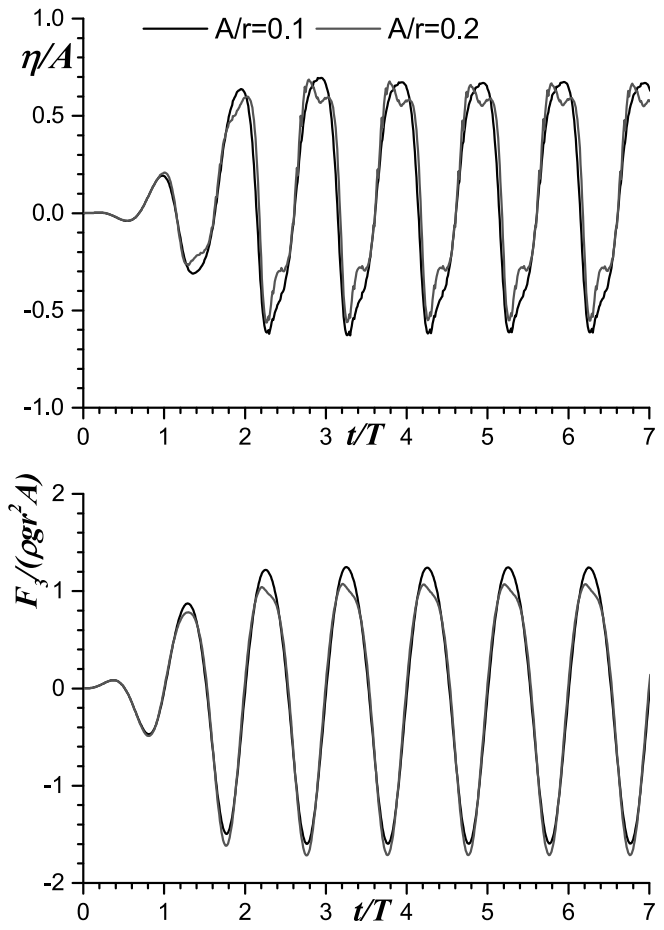

Fig. 23 Time history of wave run up and vertical force with $k r=2.294$ 

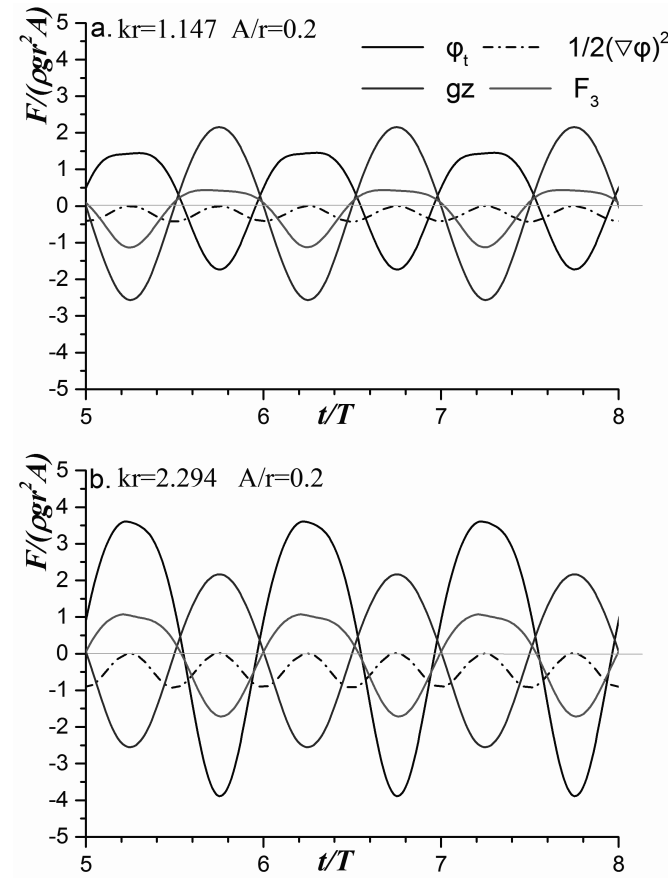

Fig. 24 Comparison of different force components; a. $k r=$ 1.47 and b. $k r=2.294$.

with well-known knowledge in the wave-body interaction theory. We can also see that the force component due to $\phi_{t}$ is opposite to the static force in phase, which is because the leading order of the force component due to $\phi_{t}$ is proportional to the acceleration of the body, see Eq. (9), and the static force is proportional to the displacement of the body.

\section{4 Wave radiation due to surge motion, case a and case $b$}

In this subsection, some of the numerical results in the forced surge motion of flared bodies are shown and the discussion is made on their features. Two different geometries i.e. case $a$ and case $b$ shown in Fig. 6 are used in the computation. Note that in case $a$ the flare angle is selected equal to $\theta=75^{\circ}$.

Figures 25 and 26 illustrate computed results for case $a$ of the wave run-up on both front side and back side and also of the forces in the horizontal and vertical directions; these are calculated at $k r=1.0$ (Fig. 25) and $k r=2.42$ (Fig. 26). Looking at the wave run-up, we can see that the phase in the time variation is almost opposite between the front side and the back side. In general, because of sinusoidal motion and symmetry of the body, a relation of $f(-x, y, z, t+T / 2)=f(x, y, z, t)$ holds, where $f$ can be the pressure or the wave elevation. Substituting this relation into the Fourierseries expansion of the vertical force $F_{3}$, one can understand that only $2 n \omega$ component exists ${ }^{16)}$. From both Fig. 25 and Fig. 26 , it can be seen that the present result for the heave force in the $z$ direction is consistent with this observation.

It should be noted that the present hydrodynamic force in heave mode is of second order and thus proportional to $A^{2}$ with increasing the amplitude of surge oscillation, which would be clear if the value will be nondimensionalized by Eq. (22). Fig. 27 illustrates the results computed for the geometry of case $b$. Despite
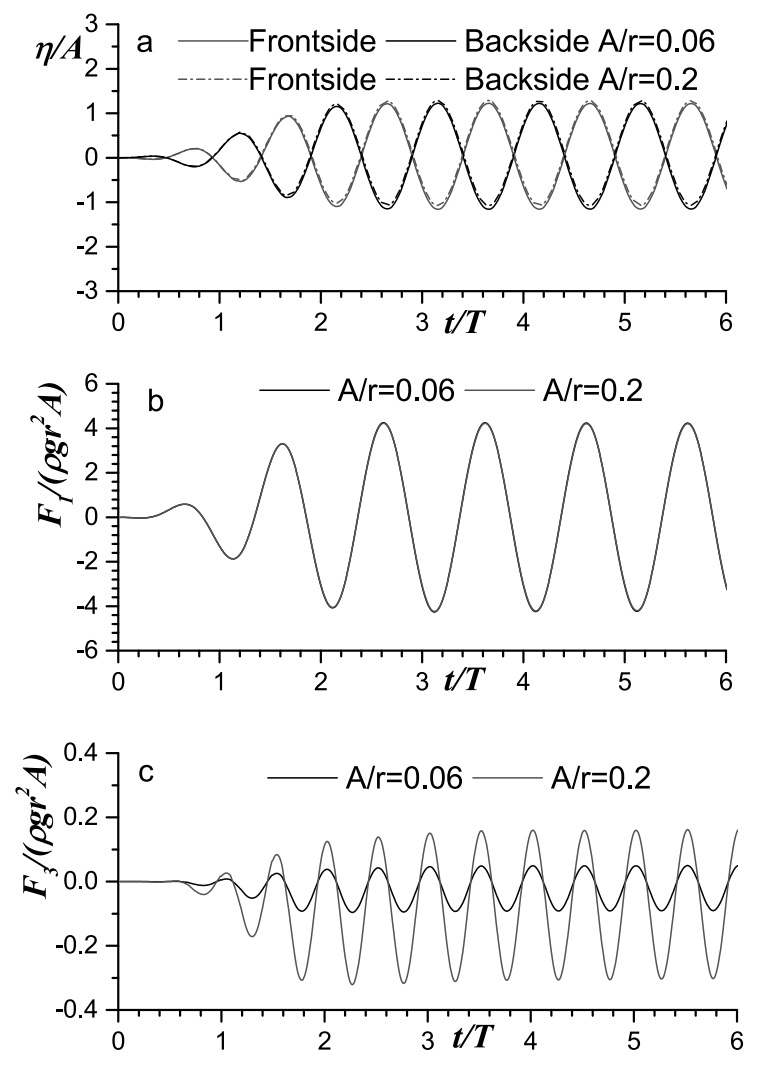

Fig. 25 Time history of wave elevations and forces on a surging body (case a) with $\theta=75^{\circ}$ and $k r=1.0$. a. wave run up; b. horizontal force; c. vertical force.

different geometry near the waterline, fundamental features in the results look the same as those for case $a$.

In the present computation, all of the nodes and the prescribed paths on the free surface move uniformly following the surge motion of a body. Thus we note that this strategy in the present ALE scheme enables us to simulate other motions with large horizontal displacement in terms of limited computational domain and mesh, for instance, the maneuvering motion of a ship.

\section{Conclusions}

The radiation problem of a 3D non-wall-sided floating body has been studied based on the fully nonlinear potential flow theory, and nonlinearity in the body-generated waves and the hydrodynamic forces on the body has been discussed. In order to solve a nonlinear free-surface problem associated with the flare of a body, Arbitrary Lagrangian-Eulerian (ALE) scheme is used in the present study. It is featured in the capability of tracking the exact intersection between the body and the water surface. Furthermore, the mesh on the free surface can be self-adapted to conform to the body motion, which is important when the body oscillates with large amplitude. In the calculation of hydrodynamic forces, a boundary-value problem for an auxiliary function related to the temporal derivative of the velocity potential $\phi_{t}$ was solved, and the accuracy and efficiency of the method have been demonstrated. From computed results, we confirmed that the flare of 

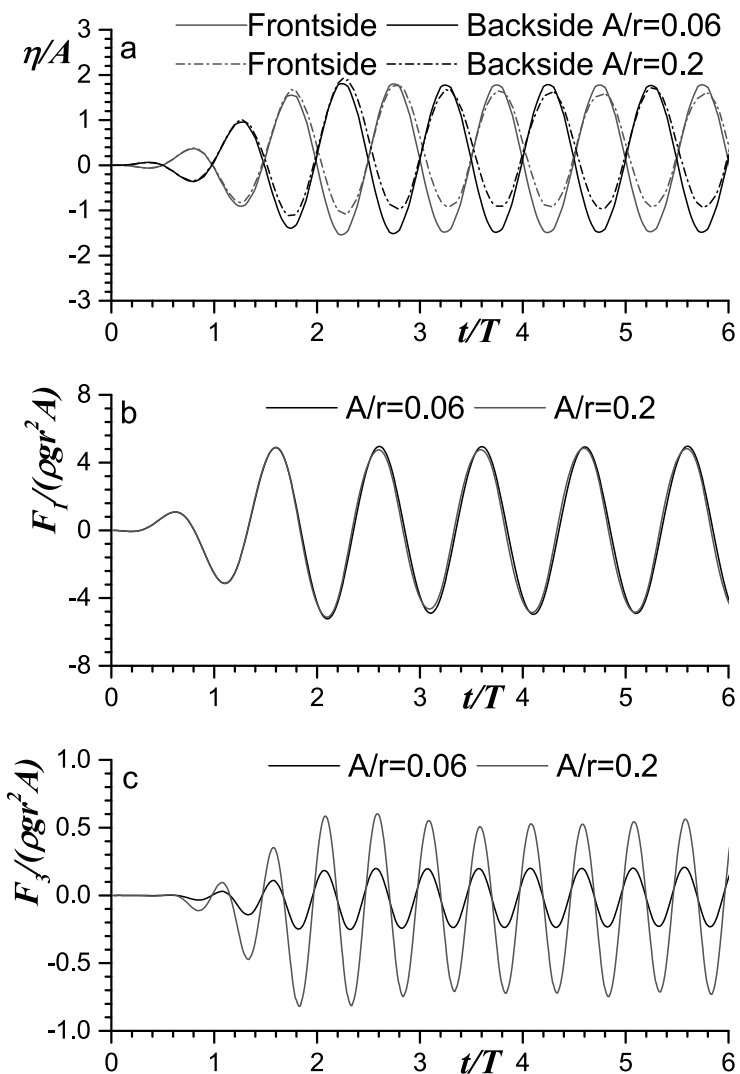

Fig. 26 Time history of wave elevations and forces on a surging body (case a) with $\theta=75^{\circ}$ and $k r=2.42$. a. wave run up; b. horizontal force; c. vertical force.

a body could increase the degree of nonlinearity in the hydrodynamic force and the wave run-up. Through various validation and confirmation for the waves and hydrodynamic forces induced by the forced oscillation of flared bodies, the calculation method presented in this paper was found to be accurate enough and flexible and hence may be applicable to more complicated problems, e.g. interaction of nonlinear waves with a freely floating body.

\section{Acknowledgments}

The first author gratefully acknowledges financial support from China Scholarship Council (CSC) (No.201406680056) and Osaka University. Special thanks also go to Mr. Zhen Yang from Technology R\&D Center, COSCO Shipping Heavy Industry CO., LTD for providing the results by HydroSTAR.

\section{References}

1) M. S. Longuet-Higgins, and E. D. Cokelet: The deformation of steep surface waves on water. I. A numerical method of computation. Proceedings of the Royal Society of London A: Mathematical, Physical and Engineering Sciences. Vol. 350. No. 1660. The Royal Society, 1976.
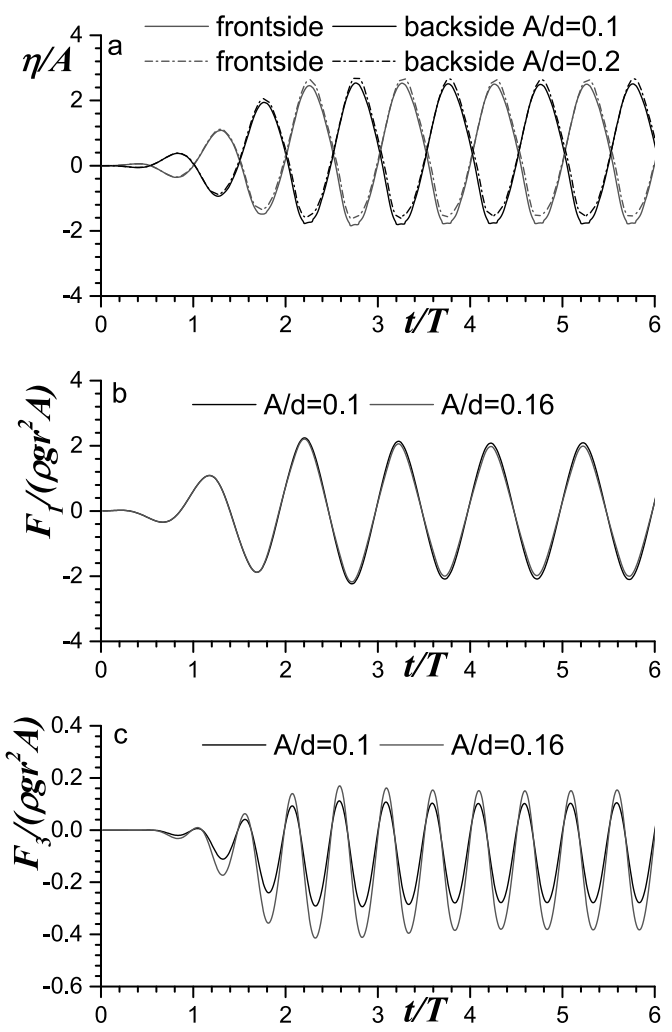

Fig. 27 Time history of wave elevations and forces on a surging body (case b) with $k r=2.04$. a. wave run up; b. horizontal force; c. vertical force.

2) Y. Shirakura, K. Tanizawa, S. Naito: Development of 3-D fully nonlinear numerical wave tank to simulate floating bodies interacting with water waves. The Tenth International Offshore and Polar Engineering Conference, 2000.

3) Y. Liu, M. Xue and D. K. Yue: Computations of fully nonlinear three-dimensional wave-wave and wav-body interactions. Part 2. Nonlinear waves and forces on a body. Journal of Fluid Mechanics, Vol.438, pp. 41-66, 2001.

4) W.C. Koo and M. H. Kim: Fully nonlinear wave-body interactions with surface-piercing bodies. Ocean Engineering, Vol.34, Num.7, pp.1000-1012, 2007.

5) J. Donea, S. Giuliani and J.P. Halleux: An arbitrary Lagrangian-Eulerian finite element method for transient dynamic fluid-structure interactions. Computer methods in applied mechanics and engineering, Vol.33, Num.1-3, pp.689723, 1982.

6) C. Zhang: Application of an improved semi-Lagrangian procedure to fully-nonlinear simulation of sloshing in nonwall-sided tanks. Applied Ocean Research, Vol.51, pp.74-92, 2015.

7) K. Tanizawa: A nonlinear simulation method of 3-D body motions in waves (1st Report). Journal of the Society of Naval Architects of Japan, Vol.178, pp.179-191, 1995.

8) M. Kashiwagi: Full-Nonlinear Simulations of Hydrodynamic Forces on a Heaving Two-Dimensional Body. Journal of the 
Society of Naval Architects of Japan, Vol.180, pp.373-381, 1996.

9) P. P. J. F. Berkvens: Floating bodies interacting with water waves: Development of a time-domain panel method, Ph.D.theses, Univ. of Twente, the Netherlands, 1998.

10) G. X. Wu and R. Eatock Taylor: Transient Motion of Floating Body in Steep Water Waves, Proc. 11 th Workshop on Water Waves and Floating Bodies, Hamburg, 1996.

11) B.Z. Zhou, D.Z. Ning, B. Teng, and W. Bai: Numerical investigation of wave radiation by a vertical cylinder using a fully nonlinear HOBEM. Vol.70, pp.1-13, 2013.

12) W. Bai, R. E. Taylor: Higher-order boundary element simulation of fully nonlinear wave radiation by oscillating vertical cylinders. Applied Ocean Research, Vol.28, Num.4, pp.247$265,2006$.

13) G. X. Wu, Z. Z. Hu: Simulation of nonlinear interactions between waves and floating bodies through a finite-elementbased numerical tank. In Proceedings of the Royal Society of London A: Mathematical, Physical and Engineering Sciences, Vol.460, No.2050, pp.2797-2817, 2004.

14) J. Zhang and M. Kashiwagi: Application of ALE to nonlinear wave diffraction by a non-wall-sided structure. In The Twenty-seventh International Offshore and Polar Engineering Conference. International Society of Offshore and Polar Engineers, San Francisco, June 25-30, 2017.

15) C. Z. Wang, G. X. Wu, and K. R. Drake: Interactions between nonlinear water waves and non-wall-sided 3D structures. Ocean Engineering, Vol.34, Num.8, pp. 1182-1196, 2007.

16) G. X. Wu: A note on non-linear hydrodynamic force on a floating body. Applied ocean research, Vol.22, Num.5, pp.315-316, 2000. 